Moreover, we also demonstrate the ability of LUM to upregulate M1 macrophage and downregulate M2 macrophage polarisation. Our findings strongly support a pathogenic role of LUM, as mediator of PAMP-induced TLR-4 activation of inflammation, cartilage degradation and macrophage polarisation in OA and other rheumatic diseases.

Disclosure of interest None declared

\section{ACPA-INDUCED MOBILITY OF PRIMED SYNOVIAL FIBROBLASTS: THE MISSING LINK BETWEEN ACPA- INDUCED BONE LOSS AND SYNOVIAL CHANGES}

${ }^{1} \mathrm{M}$ Sun*, 'B Rethi, ${ }^{1} \mathrm{~V}$ Joshua, ${ }^{1} \mathrm{~A}$ Krishnamurthy, ${ }^{1} \mathrm{~A}$ Hensvold, ${ }^{2} \mathrm{SB}$ Catrina, ${ }^{3} \mathrm{C}$ Ospelt, ${ }^{1} \mathrm{~V}$ Malmström, ${ }^{1} \mathrm{~J}$ Steen, ' $\mathrm{M}$ Engström, ${ }^{1} \mathrm{H}$ Wähämaa, ${ }^{1} \mathrm{Al}$ Catrina. ${ }^{1}$ Rheumatology unit, Department of Medicine; ${ }^{2}$ Department of Molecular Medicine and Surgery, Karolinska Institute, Stockholm, Sweden; ${ }^{3}$ Centre of Experimental Rheumatology, Department of Rheumatology, University Hospital Zürich, Zurich, Swaziland

\subsection{6/annrheumdis-2018-EWRR2018.7}

Introduction Anti-citrullinated proteins antibodies (ACPAs) injected in mice induce IL-8 dependent bone loss and arthralgia, but no synovial changes. We hypothesised that additional stimuli, sensitising the synovial compartment to ACPA effects, might be needed for the transition from bone to synovial pathology.

Methods Fibroblast like synoviocytes (FLSs) were isolated from synovial tissue biopsies obtained from RA patients. Polyclonal ACPA and other non-ACPA IgGs were separated from peripheral blood of RA patients by affinity purification on protein $G$ and cyclic citrullinated peptide (CCP) -2 columns. Monoclonal ACPAs were cloned from synovial fluid B-cells. FLS migration was tested by scratch-assays using IncuCyte (Essen bioscience). SF adhesion was analysed by xCELLigence System Real-Time Cell Analyzer (ACEA bioscience). Peptidylarginine deiminases (PAD) expression and protein citrullination were evaluated by immunohistochemistry and immunofluorescent stainings.

Results FLS mobility was not affected by polyclonal ACPA stimulation. However, exposing the cells to IL-8 or to a transient serum starvation increased PAD expression and the amount of citrullinated proteins in the cells. In line with these findings, starved FLSs obtained sensitivity to ACPAs and responded with an increased mobility to antibody stimulation. Similar effects were observed in the presence of three out of ten monoclonal ACPAs, suggesting that only APCAs with certain fine specificity could target FLSs. The ACPA-induced migration was abolished by pre-incubating the cells with PAD inhibitor or by combining the ACPAs with citrullinated but not native fibrinogen. IL-8 alone could not influence fibroblast migration but it synergistically increased the response to ACPAs. The role for inflammatory stimuli in sensitising FLSs to ACPA binding was further supported by the labelling of synovial tissues of RA patient, but not of healthy controls, using monoclonal ACPAs.

Conclusions We demonstrated that FLS response to ACPA stimulation was enabled by stress- or cytokine-induced citrullination in the cells. These results suggest an important role for transient synovial insults in setting the stage for the ACPAmediated transition towards chronic synovitis.

Disclosure of interest None declared

\section{ALTERED LYMPH NODE STROMAL CELLS DURING THE EARLIEST PHASES OF RHEUMATOID ARTHRITIS}

${ }^{1} \mathrm{C}$ Ospelt, ${ }^{1} \mathrm{E}$ Karouzakis, ${ }^{2} \mathrm{~J}$ Hähnlein, ${ }^{2} \mathrm{H}$ Semmelink, ${ }^{1} \mathrm{R}$ Gay, ${ }^{2,3} \mathrm{P}-\mathrm{P}$ Tak, ${ }^{2,4} \mathrm{D}$ Gerlag, ${ }^{1} S$ Gay, ${ }^{2} \mathrm{~L}$ Van Baarsen*. 'Center of Experimental Rheumatology, University Hospital of Zurich, Zurich, Switzerland; ${ }^{2}$ Clinical Immunology and Rheumatology, Academic Medical Centre/University of Amsterdam, Amsterdam, Netherlands; ${ }^{3}$ Current: GlaxoSmithKline, Stevenage; ${ }^{4}$ Current: Clinical Unit Cambridge, GlaxoSmithKline, Cambridge, UK

\subsection{6/annrheumdis-2018-EWRR2018.8}

Introduction Rheumatoid arthritis (RA) is an autoimmune disease with unknown etiopathogenesis where systemic autoimmunity precedes clinical disease onset. Adaptive immunity is initiated in lymphoid tissue where lymph node stromal cells (LNSC) play a crucial role in shaping the immune response and maintaining peripheral tolerance.

Objectives We developed an experimental model for studying the functional capacities of human LNSC during the earliest phases of RA and compared their cellular and molecular characteristics to LNSC from healthy volunteers.

Methods ACPA + RA patients $(\mathrm{n}=24)$, ACPA + RA risk individuals $(n=23)$ and seronegative healthy controls $(n=14 ; H C)$ underwent ultrasound-guided inguinal lymph node biopsy. Human LNSCs were isolated and expanded in vitro for cellular (flow cytometry), molecular (methylome, transcriptome and microRNA) and functional analyses.

Results Key LN chemokines CCL19, CCL21 and CXCL13 were induced in LNSCs upon stimulation with TNF $\alpha$ and lymphotoxin $\alpha 1 \beta 2$, but to a lesser extent in LNSCs from RA patients. RNA sequencing was performed on LNSC of HC $(\mathrm{n}=5)$, ACPA +RA risk individuals $(\mathrm{n}=6)$ and ACPA +RA patients $(n=4)$. Of interest, LNSC from ACPA +RA risk individuals and ACPA + RA patients revealed a common significantly differential expressed gene signature compared with $\mathrm{HC}$ LNSC. Pathway analysis of this common signature showed, among others, significant enrichment of pathways affecting actin cytoskeleton, focal adhesion and cell junction. Accordingly, in a gel contraction assay LNSC from ACPA + RA risk individuals and RA patients showed impaired collagen contraction compared to healthy LNSC. In RA LNSC a significant enrichment was observed for genes involved in TGFb signalling while in RA-risk LNSC cell cycle genes were differentially expressed compared with HC. DNA methylation analyses revealed common differentially methylated $\mathrm{CpG}$ sites (DMS) in LNSC from ACPA + RA patients $(n=5)$ and ACPA + RA risk individuals $(n=3)$ compared with HC $(n=4)$. These DMS were significantly hypomethylated and associated with antigen processing and presentation (HLA-DRB1).

Conclusions This data point towards alterations in the cytoskeleton and antigen-processing and presentation in LNSC from ACPA+RA risk individuals and RA patients. Further studies are required to investigate the influence of this LNSC abnormality on immune responses.

Disclosure of interest C. Ospelt: None declared, E. Karouzakis: None declared, J. Hähnlein: None declared, H. Semmelink: None declared, R. Gay: None declared, P.-P. Tak Employee of: Currently: Senior Vice President $\mathrm{R}$ and D Pipeline, Global Development Leader and Chief Immunology Officer, GSK, D. Gerlag Employee of: Currently: Head Clinical Unit Cambridge at GSK, S. Gay: None declared, L. Van Baarsen: None declared 